\begin{tabular}{|c|c|c|c|c|c|c|}
\hline \multirow{4}{*}{ Impact Factor: } & ISRA (India) & $=4.971$ & SIS (USA) & $=0.912$ & ICV (Poland) & $=6.630$ \\
\hline & ISI (Dubai, UAE & $=0.829$ & РИНЦ (Russia) & $=0.126$ & PIF (India) & $=1.940$ \\
\hline & GIF (Australia) & $=0.564$ & ESJI (KZ) & $=8.716$ & IBI (India) & $=4.260$ \\
\hline & JIF & $=1.500$ & SJIF (Morocco) & $=5.667$ & OAJI (USA) & $=0.350$ \\
\hline
\end{tabular}

\section{SOI: $1.1 /$ TAS $\quad$ DOI: $10.15863 /$ TAS \\ International Scientific Journal Theoretical \& Applied Science}

p-ISSN: 2308-4944 (print)

e-ISSN: 2409-0085 (online)

Year: 2020

Issue: 03

Volume: 83

Published: 26.03 .2020

\section{http://T-Science.org}

QR - Issue

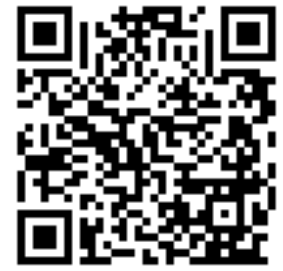

Ch.B. Normurodov

Termez state University

Ph. D., head of the Department of Applied mathematics,

(+99891) 577-93-16

normchnor2016@umail.uz

A.X. Toyirov

Termez state University doctoral student of the Department of Applied mathematics and computer science

(+99897) 242-31-50

akbartoyirov@gmail.com

Sh.M. Yuldashev

Termez state University doctoral student of the Department of Applied mathematics and computer science

(+99894) 512-48-36

shamsiddin4836@gmail.com

\title{
NUMERICAL MODELING OF NONLINEAR WAVE SYSTEMS BY THE SPECTRAL-GRID METHOD
}

\begin{abstract}
Numerical methods are increasingly used for the mathematical modeling of nonlinear wave systems. At the same time, their application to the solution of evolutionary problems with large gradients, described by nonstationary partial differential equations, is subject to serious difficulties. They are associated mainly with the presence of a small parameter with the oldest derivative and, as a consequence, the appearance in the solution of regions of strong spatial inhomogeneity. Therefore, the requirements imposed on the approximation property of numerical methods increase sharply. To solve these systems, spectral methods were mainly used. In this paper, the spectral-grid method is used to numerically simulate nonlinear wave systems. In the spectral-grid method, the interval of integration over the spatial variable is divided into a grid, in the grid elements the approximate solution is approximated with the help of a linear combination of a different number of series in Chebyshev polynomials of the first kind. Among the orthogonal polynomials, only Chebyshev polynomials have a minimax property, ie for these polynomials the maximum deviation from the required solution is minimal. In addition, for computational application of Chebyshev polynomials there are convenient recurrence formulas. With the help of these formulas it is easy to calculate the values of polynomials and their derivatives of the required order. When applying the spectral-grid method, the internal nodes of the introduced grid are subject to the continuity requirements of the approximate solution and its derivatives up to $(m-1)$-th order, where $m$ is the order of the highest derivative of the differential equation. As a result of approximation of the basic differential equation, initial-boundary conditions and continuity conditions by a spectral-grid method, a system of algebraic equations is obtained.

The spectral - grid method is applied to numerical modeling of initial - boundary value problems for heat conduction equations and nonlinear evolution equations. The numerical calculations performed show the high combining efficiency of the spectral-grid method.

Key words: mathematical modeling, nonlinear wave systems, spectral - grid method, evolution problems, interval of integration, approximation grid, Chebyshev polynomials of the first kind, algebraic system, approximate solution, efficiency, numerical results.
\end{abstract}

Language: Russian 


\begin{tabular}{|c|c|c|c|c|c|c|}
\hline \multirow{4}{*}{ Impact Factor: } & ISRA (India) & $=4.971$ & SIS (USA) & $=0.912$ & ICV (Poland) & $=6.630$ \\
\hline & ISI (Dubai, UAE & $=0.829$ & РИНЦ (Russia & $=0.126$ & PIF (India) & $=1.940$ \\
\hline & GIF (Australia) & $=0.564$ & ESJI (KZ) & $=8.716$ & IBI (India) & $=4.260$ \\
\hline & JIF & $=1.500$ & SJIF (Morocco & $=5.667$ & OAJI (USA) & $=0.350$ \\
\hline
\end{tabular}

Citation: Normurodov, C. B., Toyirov, A. X., \& Yuldashev, S. M. (2020). Numerical modeling of nonlinear wave systems by the spectral-grid method. ISJ Theoretical \& Applied Science, 03 (83), 43-54.
Soi: http://s-o-i.org/1.1/TAS-03-83-11
Doi: crossef https://dx.doi.org/10.15863/TAS.2020.03.83.11
Scopus ASCC: 2604.

\title{
ЧИСЛЕННОЕ МОДЕЛИРОВАНИЕ НЕЛИНЕЙНЫХ ВОЛНОВЫХ СИСТЕМ СПЕКТРАЛЬНО- СЕТОЧНЫМ МЕТОДОМ
}

\begin{abstract}
Аннотация: Для математического моделирования нелинейных волновых систем все более иирјго применяются численные методы. В то же время их применение к решению эволюиионных задач с большими градиентами, описываемых нестационарными уравнениями в частных производных наталькиваются на серъёзные трудности. Они связаны, главным образом, с наличием малого параметра при старшией производной и, как следствие, появлением в решении областей сильной пространственной неоднородности. Поэтому требования, предьявляемые $\kappa$ аппроксимачионным свойством численных методов, резко возрастают. Для решения указанных систем в основном применялись спектральные методы. В данной работе для численного моделирования нелинейных волновых систем применяется спектрально - сеточный метод. В спектрально-сеточном методе интервал интегрирования по пространственной переменной разбивается на сетку, в элементах сетки приближенное решение аппроксимируется с помощью линейной комбинации различного числа рядов по полиномам Чебышева первого рода. Среди ортогональных полиномов только полиномы Чебымева обладают минимаксным свойством, т.е для этих полиномов максимальное отклонение от искомого решения минимальна. Кроме того, для вычислительного применения полиномов Чебышева имеются удобные рекуррентные формулы. С помощью этих формул можно легко вычислить значения полиномов и их производных нужного порядка. При применении спектрально - сеточного метода во внутренних узлах введенной сетки налагаются требования непрерывности приближенного решения и его производных до (m-1) - го порядка, где $m$ - порядок старшей производной дифференциального уравнения. В результате аппроксимации основного дифференцииального уравнения, начально - краевых условий и условий непрерывности спектрально - сеточным методом получаются система алгебраических уравнений.

Спектрально - сеточный метод применен для численного моделирования начально - краевых задач для уравнений теплопроводности и нелинейных эволюччионных уравнений. Проведенные численные расчёты показывают высокую вычеслительную эффективности спектрально - сеточный метода.

Ключевые слова: математическое моделирование, нелинейные волновые системы, спектрально сеточный метод, эволючионные задачи, интервал интегрирования, сетка аппроксимация, полиномы Чебымева первого рода, алгебраическая система, приближенное решение, эффективность, численные результатьл.
\end{abstract}

\section{Введение}

\section{УДК 532.536}

Одним из наиболее характерных свойств волновых движений является то, что они продолжают существовать и после устранения причин, их вызывающих [1]. Волны обычно сохраняются в течение длительного времени и могут передавать возмущения на очень большие расстояния. В действительности, волны приобретают наиболее характерную для них форму именно после распространения на «большое» расстояние от области, в которой они «зародились». Одна из важных и трудных математических проблем связана с описанием поведения волн малой амплитуды, испытывающих слабую диссипацию на больших интервалах времени.

Среди нелинейных систем особое место занимают автоколебательные системы [2]. Автоколебательными системами являются часы, ламповые генераторы электромагнитных колебаний, паровые машины и двигатели внутреннего сгорания, словом, все реальные системы, которые способны совершать незатухающие колебания при отсутствии периодических воздействий извне.

Численные решения начально-краевых задач гидроаэродьнамики с большими градиентами наталкивается на серьезные трудности в связи с отсутствием достаточного пространственного разрешения в областях сильной неоднородности. Эти трудности во многих случаях преодолеваются примением спектральных методов и их модификации, обладающих свойством высокоточной пространственной аппроксимации при увеличении числа базисных функций. В прикладных расчётах, однако, не всегда удается увеличить числа базисных функций до необходимой величины, поскольку рост порядка матриц в получаемой алгебраической системы существенно ограничен ресурсами компьютеров.

В данной работе для преодоления указанных трудностей применяется спектрально-сеточный метод [3-7]. В зависимости от вида начальных данных или предполагаемого вида решения в интервале интегрирования вводятся сетка. Во внутренних узлах сетки налагается требование 


\begin{tabular}{|c|c|c|c|c|c|c|}
\hline \multirow{4}{*}{ Impact Factor: } & ISRA (India) & $=4.971$ & SIS (USA) & $=0.912$ & ICV (Poland) & $=6.630$ \\
\hline & ISI (Dubai, UAE & $=0.829$ & РИНЦ (Russia) & $=0.126$ & PIF (India) & $=1.940$ \\
\hline & GIF (Australia) & $=0.564$ & ESJI (KZ) & $=8.716$ & IBI (India) & $=4.260$ \\
\hline & JIF & $=1.500$ & SJIF (Morocco) & $=5.667$ & OAJI (USA) & $=0.350$ \\
\hline
\end{tabular}

непрерывности решения и его производных до $m-1$ порядка, где $m \quad-\quad$ порядок дифференциального уравнения. На граничных узлах сетки ставятся соответствующие краевые условия для рассматриваемой задачи. Приближенное решение на элементах сетки представляется в виде конечных рядов по полиномам Чебышева первого рода. Полученная система уравнений с помощью линейных невырожденных преобразований сводятся к двум автономным системам: линейной системе алгебраических уравнений и системе (в общем случае нелинейной) обыкновенных дифференциальных уравнений. Для решения первой системы используются стандартные методы, а для решения второй применяется явный алгоритм, развитый [16].

Поэтому применение спектрально-сеточного метода дает возможность, во-первых, распределить полиномы Чебышева по элементам с учетом поведения градиента решения и, вовторых, привести к существенному уменьшению порядка матриц в возникающий алгебраической системы. В этом методе при заданном числе элементов сетки $N$ для достижения требуемой точности расчётов необходимо правильно расположить узлы сетки и выбрать количество полиномов $p_{j}$ на элементах сетки. Эти вопросы тесно связаны, поскольку сближая узлы сетки, можно уменьшать число полиномов на элементах и наоборот. В практических расчётах более удобно выбрать равномерную сетку, задавая разные количество полиномов $p_{j}$ на каждом элементе сетки. Тогда количество необходимых полиномов зависит от относительной величины градиентов решения на том или ином элементе. Градиенты решения часто можно оценить исходя из асимптотического анализа. В задачах имеющих большие градиенты как известно [17], что вблизи стенки - в так называемом критическом слоеповедение решения опроделяется быстрым изменением вязкых решений, вдали от стенки возмущения медленно затухают.

\section{2. Постановка задачи}

Одна из важных и трудных математических проблем связана с описанием поведения волн малой амплитуды, испытывающих слабую диссипацию на больших интервалах времени [1]. Эти ограничения не такие уже специальные, как может показаться на первый взгляд. Поскольку, как следует из наблюдений, волны действительно способны долго существовать вне источников, ограничения, связанные с предположением о малой диссипации и больших интервалах времени, являются вполне естественным. В газовой динамики, имеется слабая диссипация, характеризуемая безразмерным параметром $\mathrm{Re}^{-1}$, где Re число Рейнольдса. В этом случае величина амплитуды волны $\varepsilon$ мала, но конечна. В случаях, представляющий наибольший интерес, соответствующее нелинейное уравнение следует рассматривать на интервалах времени порядка $\varepsilon^{-1}$.

Изучаемый процесс описывается уравнением Бюргерса. Рассматривается следующая начальнокраевая задача

$$
\begin{aligned}
& \frac{\partial u}{\partial t}=\frac{1}{\mu} \frac{\partial^{2} u}{\partial \eta^{2}}-u \frac{\partial u}{\partial \eta}, a<\eta<b, \\
& u(a, t)=0, \\
& u(b, t)=0, \\
& u(\eta, 0)=u_{0}(\eta) .
\end{aligned}
$$

\section{3. Спектрально-сеточный метод}

Разобьем интервал интегрирования $[a, b]$ на $M$ различных элементов:

$$
\left[\eta_{0}, \eta_{1}\right],\left[\eta_{1}, \eta_{2}\right],\left[\eta_{2}, \eta_{3}\right], \ldots,\left[\eta_{i-1}, \eta_{i}\right], \ldots,\left[\eta_{M-1}, \eta_{M}\right],
$$

где $\eta_{0}=a, \quad \eta_{M}=b$. Для представления приближенного решения в виде рядов по полиномам Чебышева каждый элемент $\left[\eta_{i-1}, \eta_{i}\right]$ интервала интегрирования $[a, b]$ отображаем на интервал $[-1,1]$ с помощью следующий замены независимой переменной

$$
\eta_{i}=\frac{m_{i}}{2}+\frac{k_{i}}{2} y,
$$

где $m_{i}=\eta_{i}+\eta_{i-1}, \quad k_{i}=\eta_{i}-\eta_{i-1}$ - длина $i$ - го элемента сетки и $y \in[-1,1]$. После этого преобразования задача (1) - (3) принимают вид:

$$
\begin{gathered}
\frac{\partial u_{i}}{\partial t}=\frac{1}{\mu}\left(\frac{2}{k_{i}}\right)^{2} \frac{\partial^{2} u_{i}}{\partial y^{2}}-\frac{2}{k_{i}} u_{i} \frac{\partial u_{i}}{\partial y} \\
i=1,2, \ldots, M, \\
u_{i}(1)=u_{i+1}(-1), \quad i=1,2, \ldots, M-1, \\
\frac{1}{k_{i}} \frac{\partial u_{i}}{\partial y}(1)=\frac{1}{k_{i+1}} \frac{\partial u_{i+1}}{\partial y}, \quad i=1,2, \ldots, M-1,(7) \\
u_{1}(-1)=u_{M}(1)=0, \\
u_{i}(y, 0)=u_{0}\left(\frac{m_{i}}{2}+\frac{k_{i}}{2} y, 0\right), i=1,2, \ldots, M,(9)
\end{gathered}
$$

где уравнения (6)-(7) требования непрерывности приближенного решения и его первой производной во внутренних узлах сетки, уравнение (8) - вид начальных данных (начальные данные для последующего положения принципиального значения не имеют и поэтому не рассматривается). 


\begin{tabular}{|c|c|c|c|c|c|c|}
\hline \multirow{4}{*}{ Impact Factor: } & ISRA (India) & $=4.971$ & SIS (USA) & $=0.912$ & ICV (Poland) & $=6.630$ \\
\hline & ISI (Dubai, UAE & $=0.829$ & РИНЦ (Russia & $=0.126$ & PIF (India) & $=1.940$ \\
\hline & GIF (Australia) & $=0.564$ & ESJI (KZ) & $=8.716$ & IBI (India) & $=4.260$ \\
\hline & JIF & $=1.500$ & SJIF (Morocce & $=5.667$ & OAJI (USA) & $=0.350$ \\
\hline
\end{tabular}

Приближенное решение уравнений (5) - (8) будем искать в виде рядов по полиномам Чебышева первого рода $T_{n}(y)$ [8-15]:

$$
\begin{array}{r}
u_{j}(y)=\sum_{n=0}^{N} a_{n}^{j} T_{n}(y), \\
T_{n}(y)=\cos (n \cdot \arccos y)
\end{array}
$$

где $N$ - количество полиномов, используемых для аппроксимации на $j$ - ом элементе. Выберем на каждом из $M$ элементов $N+1$ дискретную точку: $y_{l}=-\cos (\pi l / N), l=0,1, \ldots, N \quad$ и $\quad$ запишем систему (5)-(8) и этих точках.

$$
v \equiv\left\{u_{1}\left(y_{0}\right) . . u_{1}\left(y_{N}\right), u_{2}\left(y_{0}\right) . . u_{2}\left(y_{N}\right), u_{3}\left(y_{0}\right) \ldots, u_{M}\left(y_{0}\right) . . u_{M}\left(y_{N}\right),\right\}
$$

в $\hat{A}$ и $\hat{B}$ - квадратные матрицы размерности $((N+1) M) \times((N+1) M), \quad$ имеющие блочнодиагональную структуру:

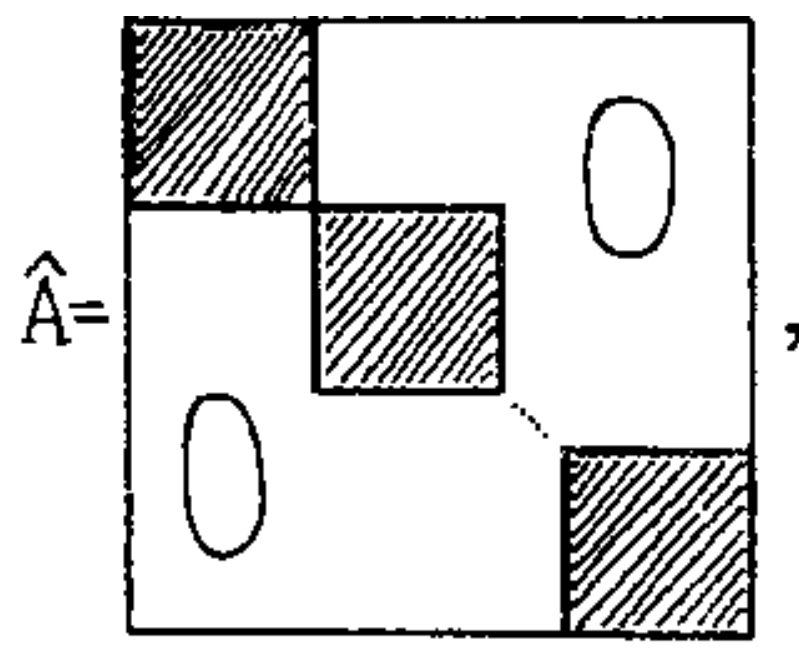

Выведем формулы для коэффициентов матриц $\hat{A}$ и $\hat{B}$.

Коэффициенты разложения $a_{m}^{j}$ для функции $u_{j}\left(y_{e}\right)$ определяются обратным преобразованием [3]:

$$
\begin{gathered}
a_{m}^{j}=\frac{2}{N C_{m}} \sum_{l=0}^{N} u_{j}\left(y_{l}\right) T_{m}\left(y_{l}\right) \\
m=0,1, \ldots, N, \quad j=1,2, \ldots, M
\end{gathered}
$$

Для этого введем матричные обозначения следующим образом. Заменим в (5)-(7) с учетом (10) производные по $y$ выражениями:

$$
\begin{aligned}
& \frac{\partial v}{\partial y}=\hat{B} v, \\
& \frac{\partial^{2} v}{\partial y^{2}}=\hat{A} v
\end{aligned}
$$

где $v$-вектор длины $(N+1) M$ с компонентами:
J

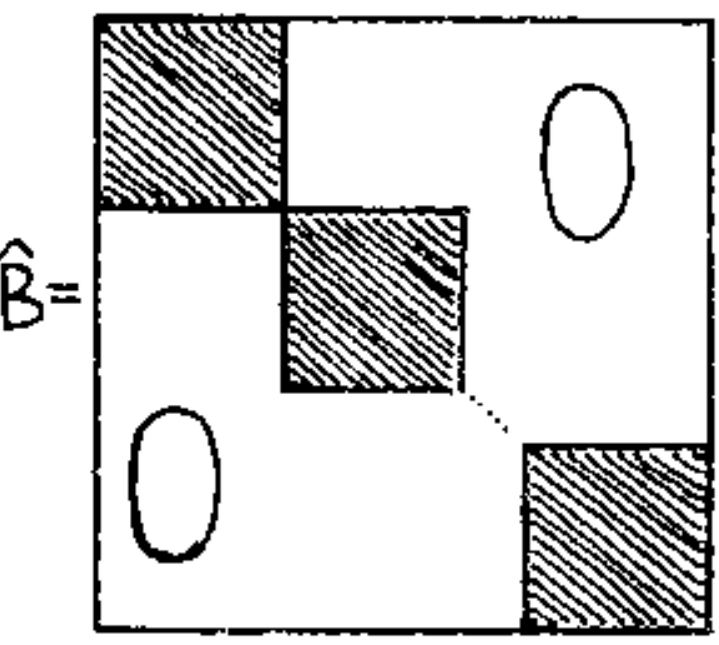

$C_{0}=C_{N}=2, C_{m}=1$, при $m \neq 0, N$.

Для удобства дальнейшего изложения запишем формулы (10) и (14) в матричном виде:

$$
\begin{aligned}
& v=T a, \\
& a=T^{*} v,
\end{aligned}
$$
где $a=\left\{a_{0}^{1}, a_{1}^{1}, \ldots, a_{N}^{1}, a_{0}^{2}, a_{1}^{2}, \ldots, a_{N}^{2}, \ldots, a_{0}^{M}, a_{1}^{M}, \ldots, a_{N}^{M}\right\}$,

- вектор (см. (13)), $T$ и $T^{*}$ - блочнодиагональные матрицы размерности $((N+1) M) \times((N+1) M):$ 


\begin{tabular}{llrllll} 
& ISRA (India) & $=\mathbf{4 . 9 7 1}$ & SIS $($ USA) & $=\mathbf{0 . 9 1 2}$ & ICV (Poland) & $=\mathbf{6 . 6 3 0}$ \\
Impact Factor: & ISI (Dubai, UAE) $=\mathbf{0 . 8 2 9}$ & PUHЦ (Russia) $=\mathbf{0 . 1 2 6}$ & PIF (India) & $=\mathbf{1 . 9 4 0}$ \\
& GIF (Australia) & $=\mathbf{0 . 5 6 4}$ & ESJI (KZ) & $=\mathbf{8 . 7 1 6}$ & IBI (India) & $=\mathbf{4 . 2 6 0}$ \\
& JIF & $=\mathbf{1 . 5 0 0}$ & SJIF (Morocco) & $=\mathbf{5 . 6 6 7}$ & OAJI (USA) & $\mathbf{0 . 3 5 0}$ \\
\hline
\end{tabular}
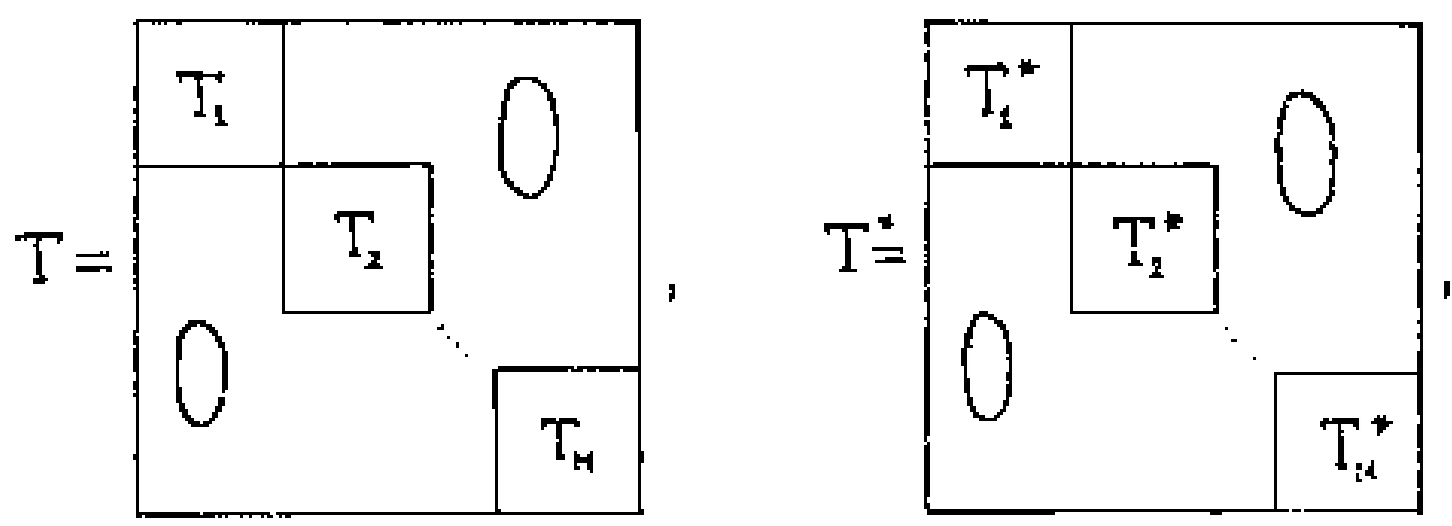

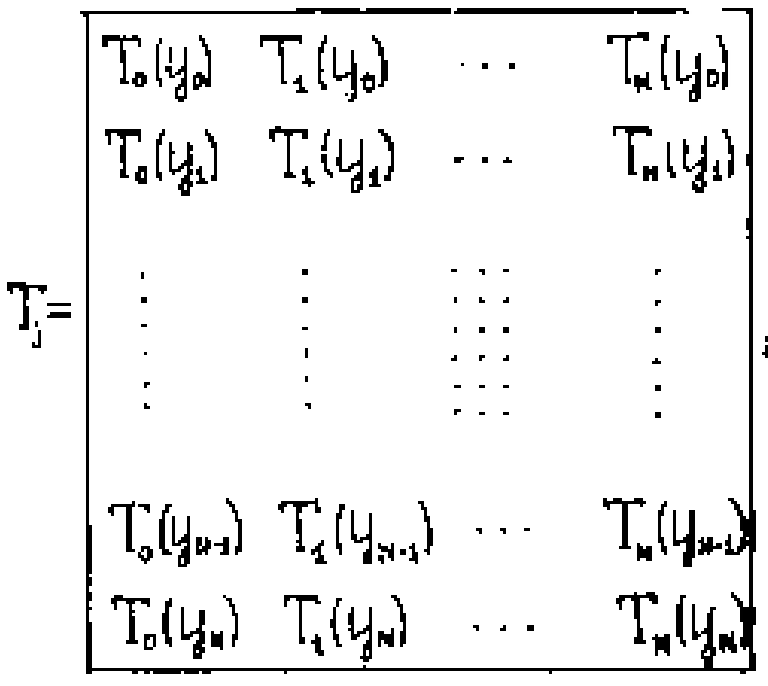

В аналогичном виде в тех же дискретных точках $y_{l}$ могут быть представлены первая и вторая пространственные производные:

$$
\begin{aligned}
& \frac{\partial v}{\partial y}=T b, \\
& \frac{\partial^{2} v}{\partial y^{2}}=T d,
\end{aligned}
$$

причем, компоненты векторов $b$ и $d$ определяются через компоненты вектора $a$ [1]

$$
\begin{aligned}
& c_{m} b_{m}^{j}=2 \sum_{\substack{P=m+1 \\
P \equiv m(\bmod 2)}}^{N} P a_{P}^{j}, \begin{array}{l}
m \geq 0 \\
j=1, \ldots, M
\end{array} \\
& c_{m} d_{m}^{j}=2 \sum_{\substack{P=m+2 \\
P \equiv m(\bmod 2)}}^{N} P\left(P^{2}-m^{2}\right) a_{P}^{j}, \begin{array}{l}
m \geq 0 \\
j=1, \ldots, M
\end{array}
\end{aligned}
$$

Запись $a \equiv b(\bmod 2)$ означает, что $a-b$ делится на 2. Формулы (19) и (20) также запишем в матричном виде:

$$
\begin{aligned}
& b=R a, \\
& d=P a .
\end{aligned}
$$

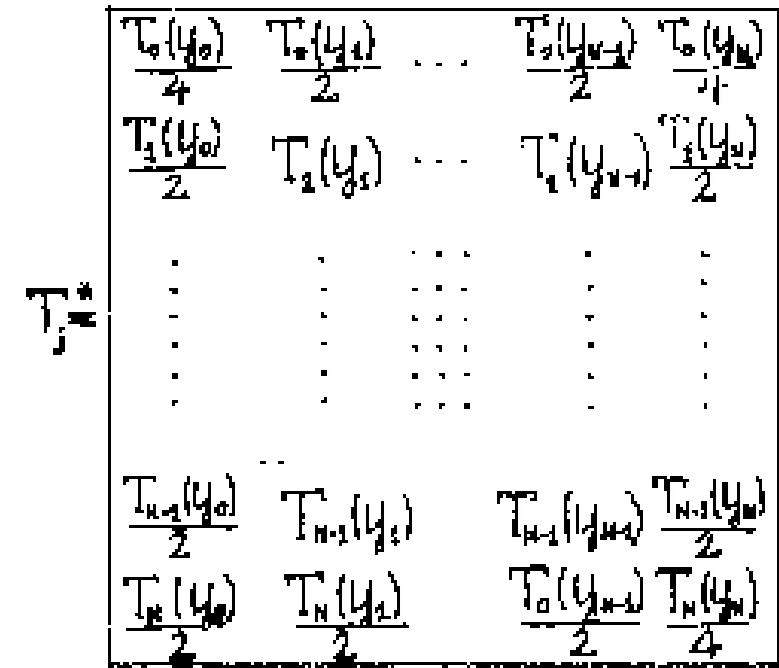

Подставляя (21) и (22) соответственно в (17) и (18) и учитывая (21), приходим к псевдоспектральной аппроксимации пространственных производных:

$$
\begin{aligned}
& \frac{\partial v}{\partial y}=\hat{B} v, \\
& \frac{\partial^{2} v}{\partial y^{2}}=\hat{A} v,
\end{aligned}
$$

где через $\hat{A}$ и $\hat{B}$ обозначены следующие матричные произведения размерности $((N+1) M) \times((N+1) M):$

$$
\hat{A}=T P T^{*}, \hat{B}=T R T^{*} \text {. }
$$

Введем также матрицы $\tilde{A}$ и $\widetilde{B}$ :

$$
\tilde{A}=\frac{1}{\mu} k^{2} \hat{A}, \tilde{B}=k \tilde{B},
$$

где $k$-вектор длины $M$ с компонентами:

$$
k \equiv\left\{k_{1}, k_{2}, k_{3}, \ldots, k_{M-1}, k_{M}\right\}
$$

Записывая дифференциальные уравнения (5) только во внутренних точках элементов $(l=1, \ldots, N-1)$, условия (6) и (7) в граничных 


\begin{tabular}{|c|c|c|c|c|c|c|}
\hline \multirow{4}{*}{ Impact Factor: } & ISRA (India) & $=4.971$ & SIS (USA) & $=0.912$ & ICV (Poland) & $=6.630$ \\
\hline & ISI (Dubai, UAE & $=0.829$ & РИНЦ (Russia & $=0.126$ & PIF (India) & $=1.940$ \\
\hline & GIF (Australia) & $=0.564$ & ESJI (KZ) & $=8.716$ & IBI (India) & $=4.260$ \\
\hline & JIF & $=1.500$ & SJIF (Morocce & $=5.667$ & OAJI (USA) & $=0.350$ \\
\hline
\end{tabular}

точках соседних элементов, а (8) в граничных точках интервала, приходим к следующей системе:

$$
\frac{d S}{d t}=A v-v \cdot B v
$$

$$
D v=0 \text {. }
$$

Здесь точка означает покомпонентное произведение двух векторов, через $S$ обозначен вектор длины $(N+1) M$ :
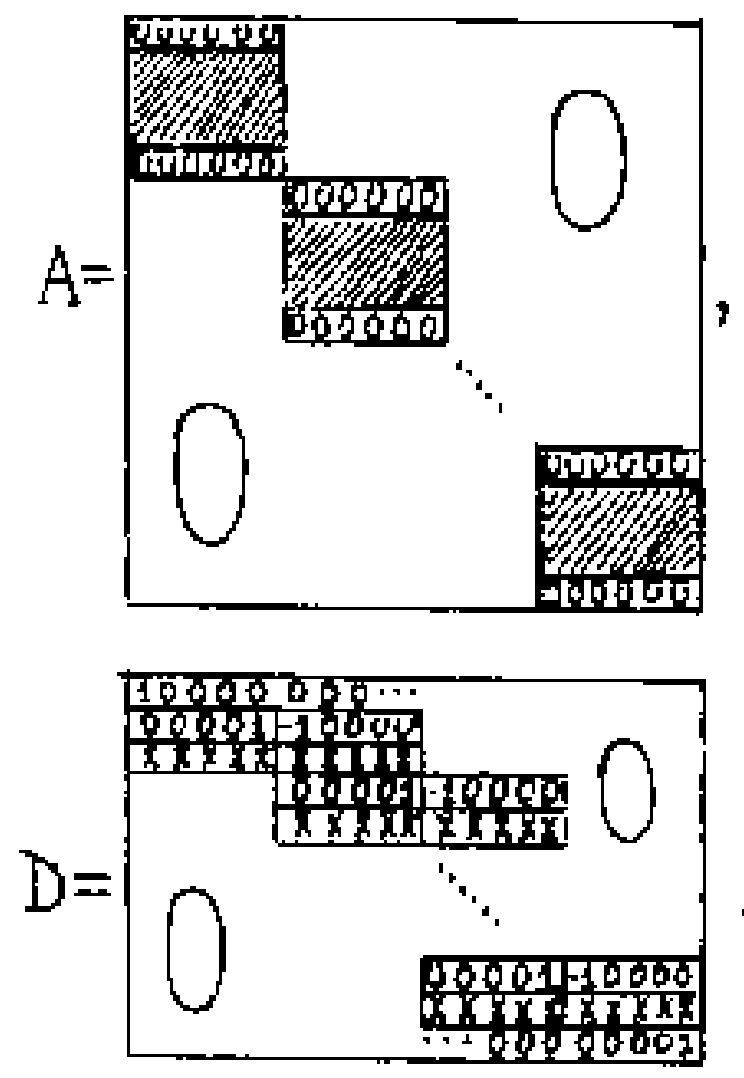

Коэффициенты каждого заштрихованного блока матриц $A$ и $B$ совпадают с соответствующими коэффициентами матриц $\tilde{A}$ и $\widetilde{B}$. Что касается матрицы $D$, то в первой и последней ее строке стоят коэффициенты условий (8), в оставшихся четных строках $(l=2 j, j=2, \ldots, M-1)$ - коэффициенты уравнений (6), а в нечетных $(l=2 j-1, j=2, \ldots, M)$ коэффициенты уравнений (29) (соответствующие строки матрицы $\widetilde{B}$ ).

$$
\text { Система (27)-(28) является }
$$

"дифференциально-алгебраической" - она содержит $(N-1) M \quad$ обыкновенных дифференциальных уравнений (26) и $2 M$ линейных алгебраических условий (28) с $(N+1) M$ неизвестными. Покажем, что путем невырожденных линейных преобразований она может быть сведена к двум автономным системам уравнений: дифференциальной системе меньшего

$$
\begin{aligned}
S \equiv & \left\{0, u_{1}\left(y_{1}\right) . . u_{1}\left(y_{N-1}\right), 0,0, u_{2}\left(y_{1}\right) . . u_{2}\left(y_{N-1}\right), 0,0,\right. \\
& \left.u_{3}\left(y_{1}\right) \ldots, 0, u_{M}\left(y_{1}\right) . . u_{M}\left(y_{N-1}\right), 0\right\},
\end{aligned}
$$

матрицы $A, \quad B \quad$ размерности $((N+1) M) \times((N+1) M)$ и матрица $D$ размерности $2 M \times((N+1) M)$ имеют вид:

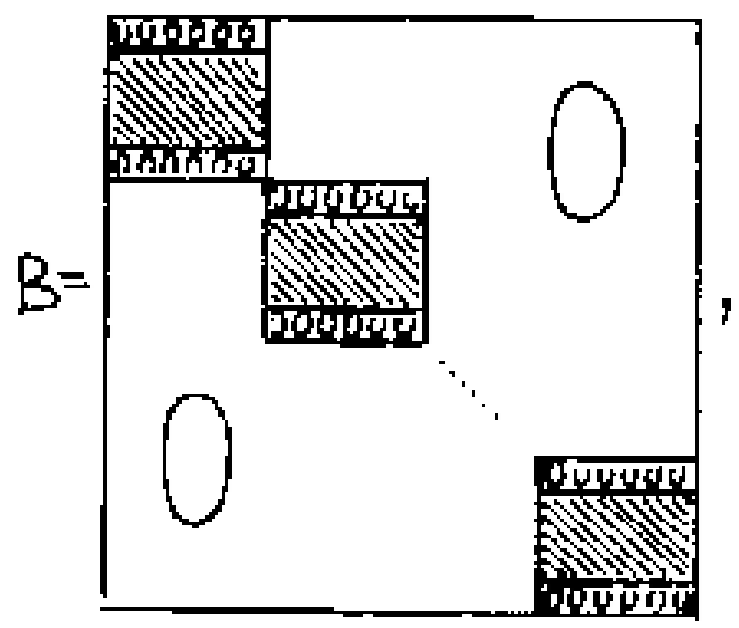

порядка $(N-1) M$ только во внутренних точках интервала и алгебраической системе стандартного вида $A x=b$ для оставшихся компонент решения (в граничных точках элементов).

Обозначим через $X, Y, V_{j}$ и $W_{j}$ комбинации переменных в условиях (27) и будем рассматривать их как новые зависимые переменные:

$$
\begin{aligned}
& V_{j} \equiv u_{j}\left(y_{N}\right)-u_{j+1}\left(y_{1}\right)=0, j=1, \ldots, M-1 \\
& W_{j} \equiv \frac{1}{K_{j}} \frac{\partial u_{j}}{\partial y}\left(y_{N}\right)-\frac{1}{K_{j+1}} \frac{\partial u_{j+1}}{\partial y}\left(y_{1}\right)=0, \\
& j=1, \ldots, M-1 \\
& X \equiv u_{1}\left(y_{0}\right)=0 \\
& Y \equiv u_{N}\left(y_{N}\right)=0
\end{aligned}
$$

тогда вектор

$$
\begin{aligned}
w= & \left\{X, u_{1}\left(y_{1}\right) . . u_{1}\left(y_{N-1}\right), V_{1}, W_{1}, u_{2}\left(y_{1}\right) \ldots u_{2}\left(y_{N-1}\right),\right. \\
& \left.V_{2}, W_{2}, \ldots, V_{M-1}, W_{M-1}, u_{M}\left(y_{1}\right) . . u_{M}\left(y_{N-1}\right), Y\right\}
\end{aligned}
$$




\begin{tabular}{|c|c|c|c|c|c|c|}
\hline \multirow{4}{*}{ Impact Factor: } & ISRA (India) & $=4.971$ & SIS (USA) & $=0.912$ & ICV (Poland) & $=6.630$ \\
\hline & ISI (Dubai, UAE & $=0.829$ & РИНЦ (Russia) & $=0.126$ & PIF (India) & $=1.940$ \\
\hline & GIF (Australia) & $=0.564$ & ESJI (KZ) & $=8.716$ & IBI (India) & $=4.260$ \\
\hline & JIF & $=1.500$ & SJIF (Morocco) & $=5.667$ & OAJI (USA) & $=0.350$ \\
\hline
\end{tabular}

совпадает с $S$ из (30) и связан с $v$ из (13) соотношением:

$$
w=G v .
$$

Здесь $G$ - невырожденная матрица размерности $((N+1) M) \times((N+1) M)$

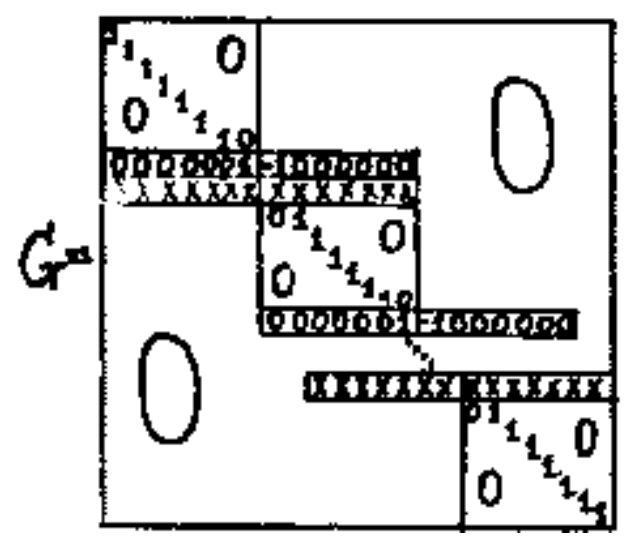

в которой строки с номерами $j(N+1)(j=1, \ldots, M-1)$ суть строки матрицы $D$ с номерами $2 j(j=1, \ldots, M-1), \quad$ а строки $j(N+1)+1(j=1, \ldots, M-1)-\quad$ ее строки с номерами $2 j+1$.

Введем также обозначения:

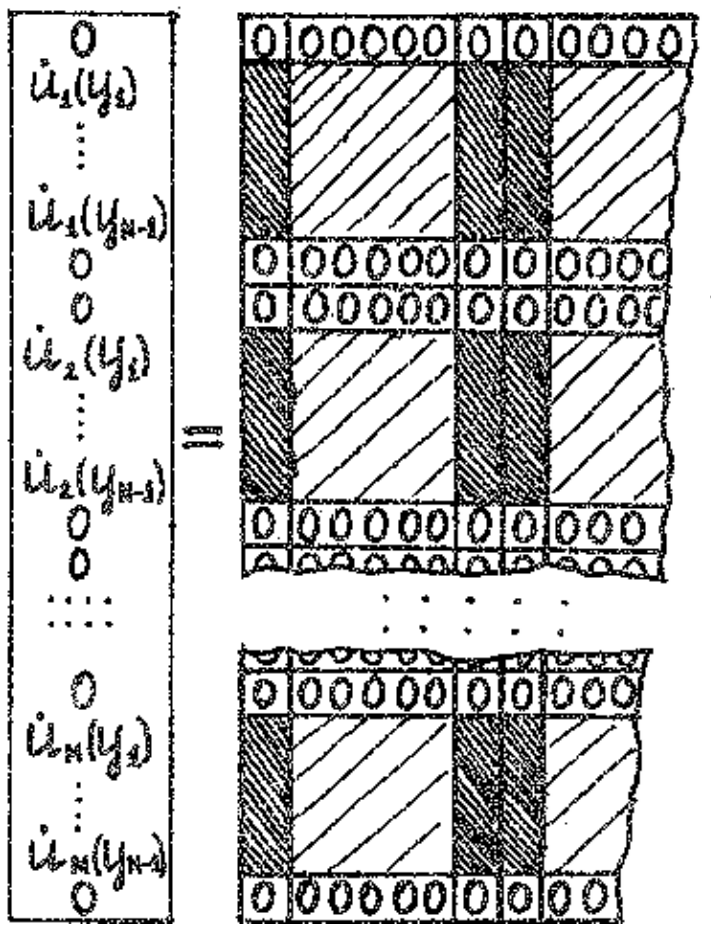

Таким образом, из (35а) видно, что система обыкновенных дифференциальных уравнений (26) свелась к системе меньшей размерности, причем с невырожденной матрицей $H$ (из матрицы $\hat{H}$ исключаются все нулевые строки и все столбцы, элементы которых умножаются на соответствующие нулевые элементы вектора $w$;

$$
\begin{aligned}
& \hat{H}=A G^{-1}, \\
& \hat{f}=v \cdot B v,
\end{aligned}
$$

в которых (26) принимает вид:

$$
\frac{d w}{d t}=\hat{H} w-\hat{f}
$$

или покомпонентно:
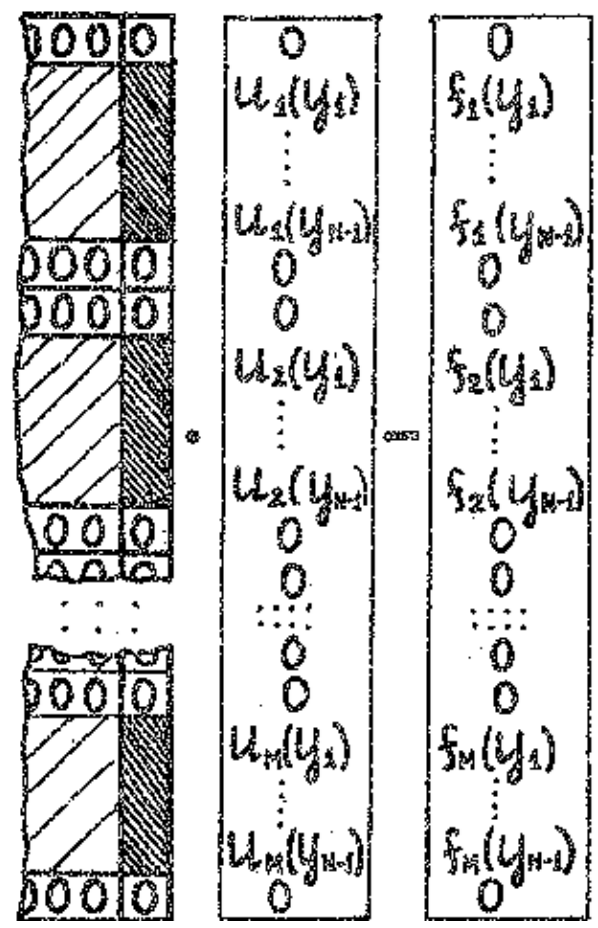

(35a)

несколько таких столбцов выделено на рис.(35a) штриховкой):

$$
\frac{d r}{d t}=H r-f
$$

Здесь

$H:(M(N-1)) \times(M(N-1))$, a 


\begin{tabular}{|c|c|c|c|c|c|c|}
\hline \multirow{4}{*}{ Impact Factor: } & ISRA (India) & $=4.971$ & SIS (USA) & $=0.912$ & ICV (Poland) & $=6.630$ \\
\hline & ISI (Dubai, UAE & $=0.829$ & РИНЦ (Russia) & $=0.126$ & PIF (India) & $=1.940$ \\
\hline & GIF (Australia) & $=0.564$ & ESJI (KZ) & $=8.716$ & IBI (India) & $=4.260$ \\
\hline & JIF & $=1.500$ & SJIF (Morocco) & $=5.667$ & OAJI (USA) & $=0.350$ \\
\hline
\end{tabular}

$$
\begin{aligned}
r= & \left\{u_{1}\left(y_{1}\right) . . u_{1}\left(y_{N-1}\right), u_{2}\left(y_{1}\right) \ldots u_{2}\left(y_{N-1}\right), \ldots\right. \\
& \left., u_{M}\left(y_{1}\right) . . u_{M}\left(y_{N-1}\right)\right\}, \\
f= & \left\{f_{1}\left(y_{1}\right) \ldots f_{1}\left(y_{N-1}\right), f_{2}\left(y_{1}\right) \ldots f_{2}\left(y_{N-1}\right), \ldots\right. \\
& \left., f_{M}\left(y_{1}\right) \ldots f_{M}\left(y_{N-1}\right)\right\}
\end{aligned}
$$

- векторы длины $(M(N-1))$, причем, $r$ вектор отличается от вектора $v$ только отсутствием компонент с номерами $(j-1) N+1$ и $j N$, где $j=1, \ldots, M$. Недостающие компоненты вектора $v$ находятся путем решения линейной алгебраической системы (32).

Система уравнений (36) является эволюционной. Для ее численного решения в данной работе, как уже говорилось, применялся метод [16,17] Именно, для перехода на следующий временной слой использовалась явная схема вида:

$$
\begin{aligned}
& r(t+\tau)=r(t)+R Q r(t)+R f(t), \\
& R \varphi(t)=\frac{\tau}{12}[23 \varphi(t)-16 \varphi(t-\tau)+5 \varphi(t-2 \tau)],(37) \\
& Q=\frac{12\left(e^{H \tau}-E\right)}{\tau\left(23 E-16 e^{-H \tau}+5 e^{-2 H \tau}\right)},
\end{aligned}
$$

где матрица $Q$ получена с помощью специальных преобразований системы (36) - см. [16], $R$ оператор схемы Адамса-Бэшфорта 3-его порядка, $E$ - единичная матрица, $\tau$ - шаг интегрирования. Применение схемы (37) в сравнении с обычной схемой Адамса-Бэшфорта дает возможность существенно ослабить ограничения на $\tau$, связанные с требованиями ее устойчивости.

Таким образом, последовательность вычислений следующая:

1) область интегрирования разбивается на некоторое число элементов $M$;

2) одношаговым алгоритмом (например, методом Рунге-Кутта) находится решение на первых двух временных слоях: $t=\tau, t=2 \tau$;

3) осуществляется ряд невырожденных преобразований матрицы $H$ с целью ослабления условия устойчивости;

4) по формуле (37) осуществляется переход на новый временной слой $t=\tau$;

5) компоненты вектора $v$ в граничных точках элементов находятся из решения алгебраической системы (32).

\section{4. Результаты расчётов}

Построенный алгоритм был применен к расчетам одномерных начально-краевых задач для уравнений теплопроводности и Бюргерса.

В случае уравнения теплопроводности:

$$
\frac{\partial u}{\partial t}=\frac{1}{\mu} \frac{\partial^{2} u}{\partial \eta^{2}},
$$

проводилось сравнение с аналитическим решением по нормам ошибок:

абсолютной:

$$
\Delta=\max _{j} \mid u_{r}\left(\eta_{j}, t_{\phi}\right)-u_{\tau}\left(\eta_{j}, t_{\phi}\right),
$$

и относительной:

$$
\varepsilon=\frac{\Delta}{\max _{j}\left|u_{r}\left(\eta_{j}, t_{\phi}\right)\right|},
$$

где $u_{r}\left(\eta, t_{\phi}\right)$ и $u_{\tau}\left(\eta, t_{\phi}\right)$ - соответственно численное и аналитическое решение в фиксированный момент $t_{\phi}$. Начальные условия в момент $t=0$ выбирались в виде гауссова распределения, нормированного на 1 . Точное аналитическое решение в этом случае имеет вид:

$$
\begin{aligned}
& u_{r}(\eta, t)=\sqrt{\frac{t_{0}}{t+t_{0}}} \cdot \exp \left[-\frac{\eta^{2}}{4 R\left(t+t_{0}\right)}\right], \\
& R=1 / \mu, \eta \in]-\infty, \infty[
\end{aligned}
$$

Постоянная $t_{0}$ определяет полуширину начального распределения:

чем меньше $t_{0}$, тем она уже. Всюду в расчетах: $t_{0}=0.15, \quad \mu=\pi \cdot 10^{2}, n=32, \tau=0.01$. Интервал интегрирования выбран $[-1,1]$. При заданной полуширине начального распределения функция (41) в граничных точках равна нулю с точностью $10^{-12}$. Поэтому уравнение (41) решалось при следующих граничных условиях:

$$
u( \pm 1, t)=0,
$$




\begin{tabular}{|c|c|c|c|c|c|c|}
\hline \multirow{4}{*}{ Impact Factor: } & ISRA (India) & $=4.971$ & SIS (USA) & $=0.912$ & ICV (Poland) & $=6.630$ \\
\hline & ISI (Dubai, UAE & $=0.829$ & РИНЦ (Russia & $=0.126$ & PIF (India) & $=1.940$ \\
\hline & GIF (Australia) & $=0.564$ & ESJI (KZ) & $=8.716$ & IBI (India) & $=4.260$ \\
\hline & JIF & $=1.500$ & SJIF (Morocce & $=5.667$ & OAJI (USA) & $=0.350$ \\
\hline
\end{tabular}

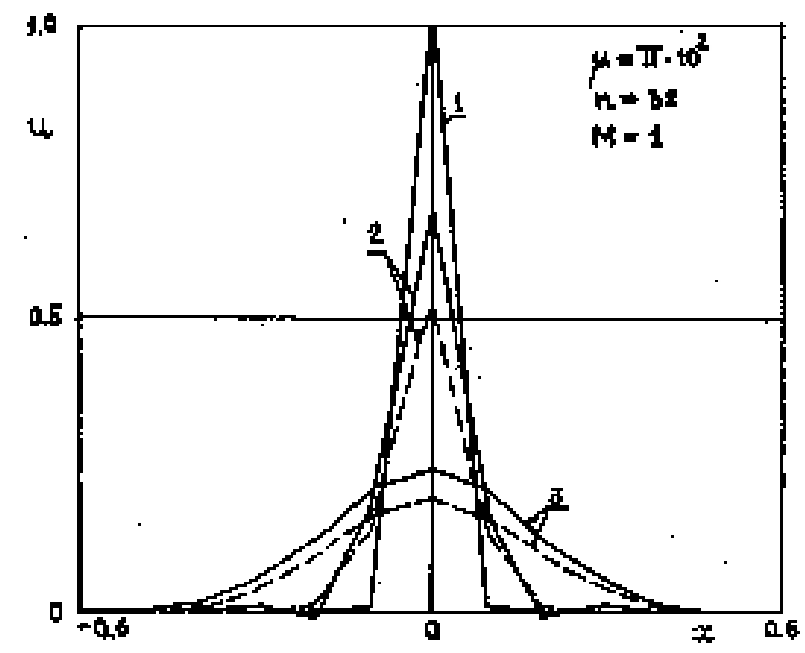

Рис.1 - эволюция численного решения

На рис.1 показана эволюция численного решения задачи (38), (42), найденного с использованием построенного метода в случае, когда область интегрирования не разбивалась на элементы $(M=1)$. Сплошные кривые 1, 2, 3 соответствуют численному решению в моменты времени $t=0,40 \tau, 400 \tau$. Пунктирные кривые 2,3 соответствуют точному решению (41) в те же моменты времени. Видно, что при использовании полиномов Чебышева узлы на интервале расположены не рационально - они сгущаются к краям интервала, в то время как в области быстрого изменения решения находится всего лишь $14 \%$ узлов. В результате точность решения оказывается низкой:

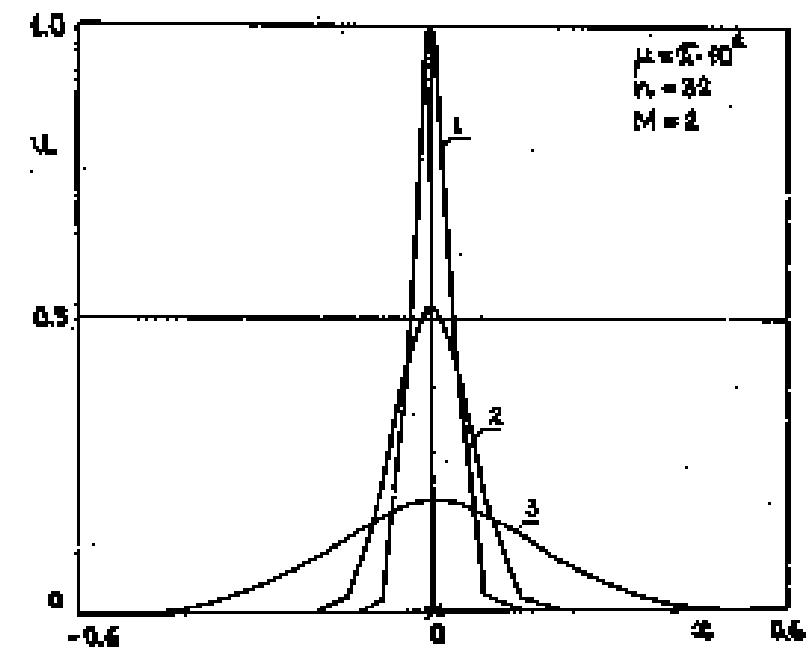

Рис.2 - эволюцию численного решения

Рис. 2 показывает эволюцию численного решения той же задачи для случая, когда область интегрирования разбивалась на 2 элемента: $[-1,0],[0,1]$. Такое разбиение является более удачным - в область неоднородности попадает около $30 \%$ узлов сетки. Точность в этом случае на 2 порядка выше (см. табл. 2), поэтому сплошные кривые (численное решение) и пунктирные (точное решение) на рис. практически неразличимы. 


\begin{tabular}{|c|c|c|c|c|c|c|}
\hline \multirow{4}{*}{ Impact Factor: } & ISRA (India) & $=4.971$ & SIS (USA) & $=0.912$ & ICV (Poland) & $=6.630$ \\
\hline & ISI (Dubai, UAE & $=0.829$ & РИНЦ (Russia) & $=0.126$ & PIF (India) & $=1.940$ \\
\hline & GIF (Australia) & $=0.564$ & ESJI (KZ) & $=8.716$ & IBI (India) & $=4.260$ \\
\hline & JIF & $=1.500$ & SJIF (Morocco) & $=5.667$ & OAJI (USA) & $=0.350$ \\
\hline
\end{tabular}

Таблица 1. Результаты расчетов

\begin{tabular}{|c|c|c|c|c|c|}
\hline$t$ & 0.01 & 0.1 & 0.4 & 2.0 & 4.2 \\
\hline$\Delta$ & $2 \cdot 10^{-2}$ & $10^{-1}$ & $2 \cdot 10^{-1}$ & $9 \cdot 10^{-2}$ & $6 \cdot 10^{-2}$ \\
\hline$\varepsilon$ & $2 \cdot 10^{-2}$ & $10^{-1}$ & $3 \cdot 10^{-1}$ & $3 \cdot 10^{-1}$ & $2 \cdot 10^{-1}$ \\
\hline
\end{tabular}

Таблица 2. Результаты расчетов

\begin{tabular}{|c|c|c|c|c|c|}
\hline$t$ & 0.01 & 0.1 & 0.4 & 2.0 & 4.2 \\
\hline$\Delta$ & $2 \cdot 10^{-2}$ & $5 \cdot 10^{-3}$ & $2 \cdot 10^{-3}$ & $7 \cdot 10^{-4}$ & $5 \cdot 10^{-4}$ \\
\hline$\varepsilon$ & $2 \cdot 10^{-2}$ & $6 \cdot 10^{-3}$ & $4 \cdot 10^{-3}$ & $3 \cdot 10^{-3}$ & $2 \cdot 10^{-3}$ \\
\hline
\end{tabular}

Аналогичный эффект можно наблюдать и в случае нелинейных эволюционных уравнений. Ниже рассматривается начально-краевая задача для уравнения Бюргерса:

$$
\begin{aligned}
& \frac{\partial u}{\partial t}=\frac{1}{\mu} \frac{\partial^{2} u}{\partial \eta^{2}}-u \frac{\partial u}{\partial \eta}, \\
& u(\eta, 0)=-\sin \pi \eta, \\
& u( \pm 1, t)=0 .
\end{aligned}
$$

Аналитическое решение задачи (43) - (44) имеет вид отношения рядов из функций Бесселя [16]. При больших числах $\mu\left(\mu \geq 10^{2}\right)$ сравнение численного решения задачи (43)-(44) с аналитическим затруднено ввиду медленной сходимости рядов в аналитическом решении [16]. Поэтому точность вычислений оценивалась по порядку величины последних коэффициентов $a_{n}^{j}$ в разложении (10) (порядок ошибки не превосходит указанной величины).

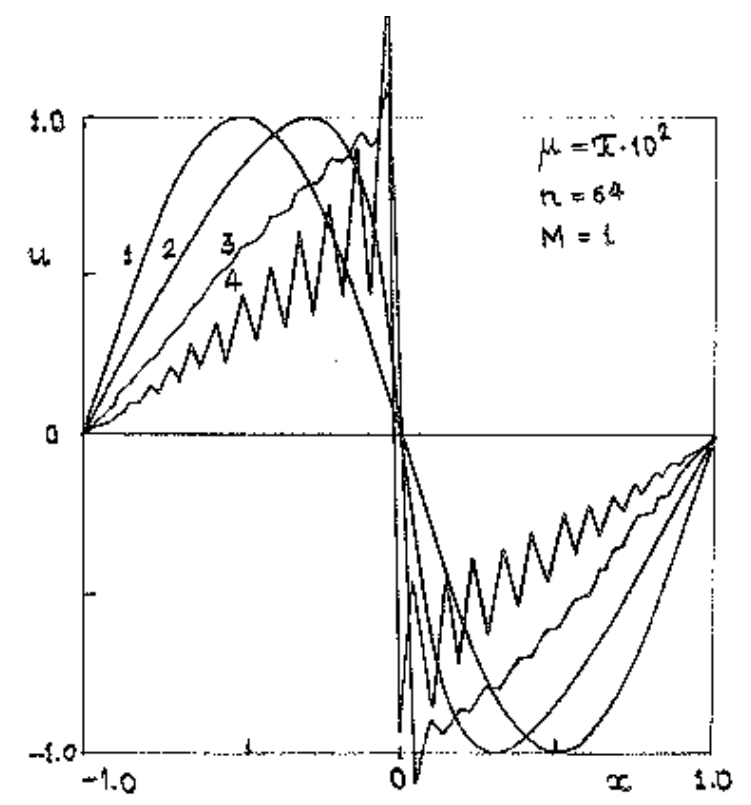

Puc.3
Конкретные вычисления были проведены при значениях параметров: $\mu=\pi \cdot 10^{2}, n=64, \tau=10^{-2} / 6 \pi . \quad$ Результаты представлены на рис.3 и 4. На обоих рисунках кривые 1,2,3,4 показывают поведение численного решения, в момент времени $t=0 ., 0.2,0.5,1.0$, а кривая 5 на рис. 4 соответствует моменту $t=2.0$. На рис.3 $(M=1)$ видно, что с ростом первой производной в точке $\eta=0$ численное решение начинает осциллировать и при $t=1.0$ амплитуда осцилляций вырастает настолько, что в решении не остается ни одного верного знака. В случае разбиения области интегрирования на 2 элемента: $[-1,0],[0,1]$ (рис.4) амплитуда осцилляций при $t=0.5$ существенно меньше - решение имеет 2 верных знака. Когда $t>0.5$ осцилляции сглаживается, точность решения вновь возрастает - к моменту $t=2.0$, например, она достигает величины $\varepsilon \sim 10^{-4}$.

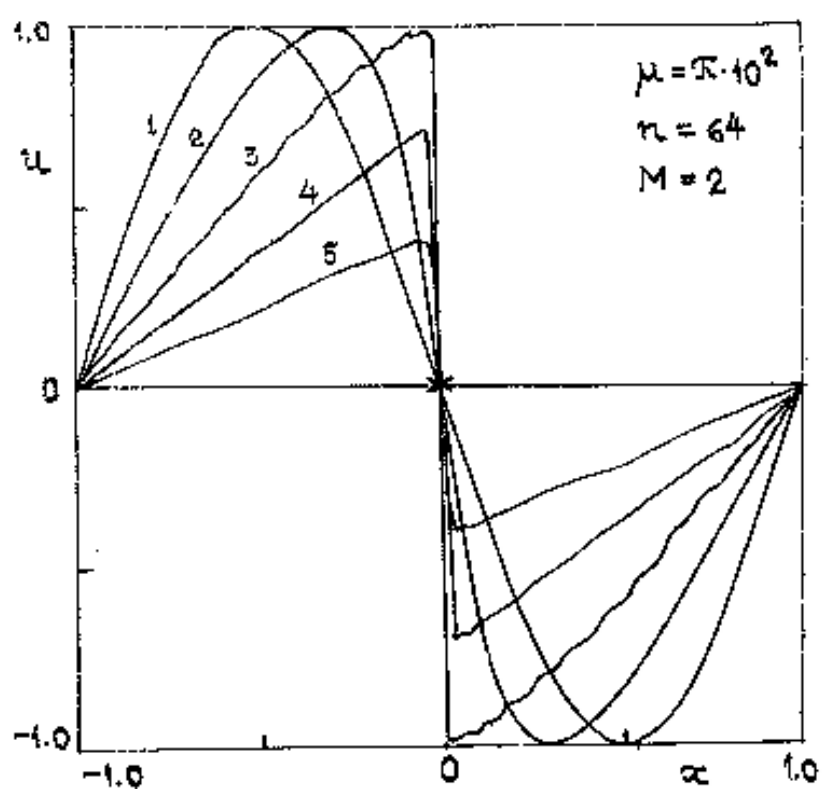

Puc. 4 


\begin{tabular}{|c|c|c|c|c|c|c|}
\hline \multirow{4}{*}{ Impact Factor: } & ISRA (India) & $=4.971$ & SIS (USA) & $=0.912$ & ICV (Poland) & $=6.630$ \\
\hline & ISI (Dubai, UAE & $=0.829$ & РИНЦ (Russia) & $=0.126$ & PIF (India) & $=1.940$ \\
\hline & GIF (Australia) & $=0.564$ & ESJI (KZ) & $=8.716$ & IBI (India) & $=4.260$ \\
\hline & JIF & $=1.500$ & SJIF (Morocco) & $=5.667$ & OAJI (USA) & $=0.350$ \\
\hline
\end{tabular}

На рис.5 показана эволюция численного решения для случая, когда интервал интегрирования разбит на большее число элементов (M=8). Значения всех параметров те же, что и в предыдущих расчетах. Длины элементов соотносятся следующим образом:

$$
K_{1}: K_{2}: K_{3}: K_{4}=8: 4: 2: 1 ; K_{5}=K_{4}, K_{6}=K_{3},
$$
$K_{7}=K_{2}, K_{8}=K_{1}$. Видно, что при всех $\eta$ за исключением узкой области вблизи $\eta=0$, где амплитуда осцилляций пренебрежимо мала, осцилляции отсутствуют для всех значений $t$.

Результаты расчетов, таким образом, показывают, что за чет разбиения интервала интегрирования ка элементы точность численного решения в области больших градиентов может быть существенно повышена.
Представляет также определенный интерес сравнение изложенного метода с методом, описанным в [16]. Как и в данной работе в [16] использована спектрально-сеточная аппроксимация пространственных производных, причем на элементах решение аппроксимировалось рядами по полиномам Чебышева. В табл. 3 приведена величина $\max \left|\frac{\partial u}{\partial \eta}\right|$ , вычисленная обоими методами, а также точное значение этой величины, найденное из аналитического решения.

Через $t_{\max }$ здесь обозначен момент времени, на который

Таблица 3. Результаты расчетов (здесь $\mu=100 \pi$ )

\begin{tabular}{|l|l|l|l|l|l|}
\hline Метод & интервал & $\left|\frac{\partial u}{\partial \eta}\right|_{\max }$ & $\pi t_{\max }$ & $N \times M$ & $\pi \tau$ \\
\hline $\begin{array}{l}\text { 1.Спектрально- сеточный } \\
\text { метод (данная работа) }\end{array}$ & {$[-1,1]$} & 152.4 & 1.6038 & $16 \times 4$ & $10^{-2 / 6}$ \\
\hline $\begin{array}{l}\text { 2.Спектрально-элементный } \\
\text { (Ранега. А.Т [9]) }\end{array}$ & {$[-1,1]$} & 152.0 & 1.6033 & $16 \times 4$ & $10^{-2 / 6}$ \\
\hline 3.Аналитическое решение & & 152.0 & 1.6037 & & \\
\hline
\end{tabular}

достигает максимума. Значения всех параметров в обоих случаях совпадают. Видно, что и в том и в другом случае точность расчетов имеет одинаковый порядок. Преимущество предлагаемого метода однако, заключается в большей общности, поскольку в отличие от [18] в нем не содержится каких-либо предположений о форме решения, а следовательно не требуется введения, дополнительных параметров, определяемых в процессе расчета.

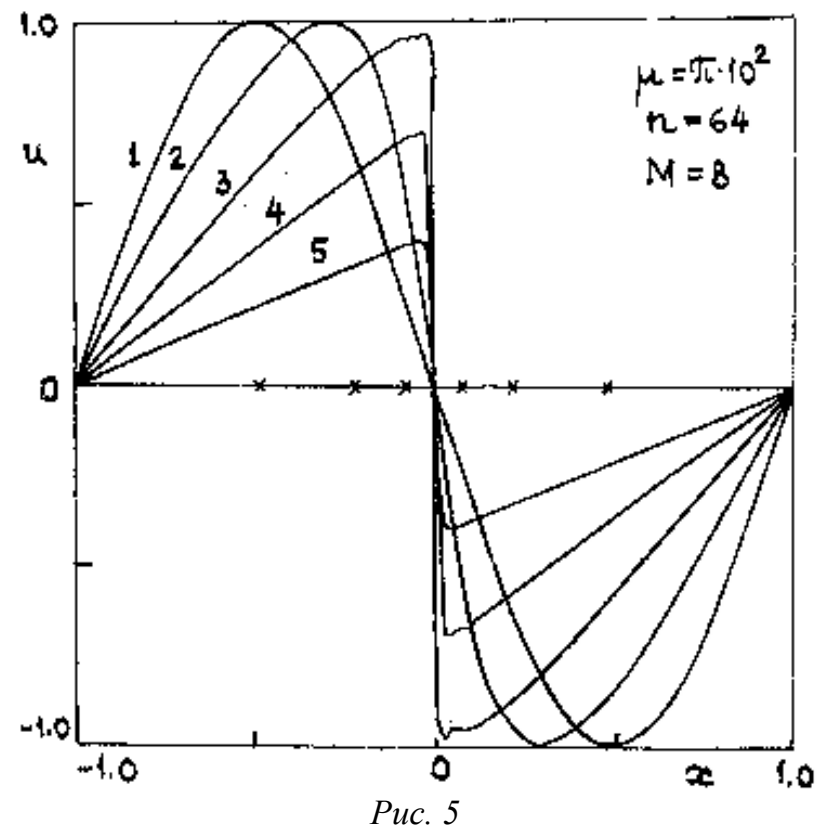




\begin{tabular}{|c|c|c|c|c|c|c|}
\hline \multirow{4}{*}{ Impact Factor: } & ISRA (India) & $=4.971$ & SIS (USA) & $=0.912$ & ICV (Poland) & $=6.630$ \\
\hline & ISI (Dubai, UAE & $=0.829$ & РИНЦ (Russia & $=0.126$ & PIF (India) & $=1.940$ \\
\hline & GIF (Australia) & $=0.564$ & ESJI (KZ) & $=8.716$ & IBI (India) & $=4.260$ \\
\hline & JIF & $=1.500$ & SJIF (Morocce & $=5.667$ & OAJI (USA) & $=0.350$ \\
\hline
\end{tabular}

\section{5. Заключение}

1. Построен спектрально-сеточный явный метод решения эволюционных задач с большими градиентами. В зависимости от местоположения областей неоднродности интервал интегрирования разбивается на конечное число элементов. На каждом из элементов используется спектральная аппроксимация конечными рядами по базисным функциям.
2. Выполнены конкретные расчеты для одномерного уравнения Бюргерса, проведено сравнение с одноэлементным псевдоспектральным методом.

3. Показано, что применение спектральносеточной аппроксимации дает возможность значительно повысить точность расчетов, не увеличивая общее число базисных функций.

\section{References:}

1. Lejbovicha, C., \& Sibassa, A. (1977). Nelinejnye volny. (p.320). Moscow: Mir.

2. Butenin, N.V., Nejmark, Ju.I., \& Fufaev, N.A. (1976). Vvedenie $v$ teoriju nelinejnyh kolebanij. (p.384). Moscow: Nauka.

3. Narmuradov, Ch.B. (2005). Ob odnom jeffektivnom metode reshenija uravnenija OrroZommerfel'da. Matematicheskoe modelirovanie, Moskva, № 9 (17), pp. 35-42.

4. Narmuradov, Ch.B. (2007). Matematicheskoe modelirovanie gidrodinamicheskih zadach dlja dvuhfaznyh ploskoparallel'nyh techenij. Matematicheskoe modelirovanie, Moskva, № 6 (19), pp. 53-60.

5. Abutaliev, F.B., \& Narmuradov, Ch.B. (2011). Matematicheskoe modelirovanie problemy gidrodinamicheskoj ustojchivosti. (p.188). Tashkent: «Fan va texnologiya».

6. Narmuradov, Ch.B. (2001). Algoritm spektral'no-setochnogo metoda dlja reshenija zadachi gidrodinamicheskoj ustojchivosti pogranichnogo sloja. Uzbekskij zhurnal "Problemy informatiki i jenergetiki», Tashkent, № 5-6, pp. 57-60.

7. Narmuradov, Ch.B. (2004). Ob jeffektivnom metode reshenija zadachi gidrodinamicheskoj ustojchivosti dlja dvuhfaznyh potokov. Dokl. AN $R U z$., Tashkent, № 1, pp. 19-26.

8. Pashkovskij, S. (1983). Vychislitel'nye primenenija mnogochlenov $i$ rjadov Chebysheva. Moscow: Nauka.

9. Basdevant, C., et al. (1986). Spectral and finitedifference solutions of the Burgers equation. Computers \& Fluids, V. 14, pp. 23-41.
10. Orszag, S.A. (1971). Numerical simulation of incompressible flows within simple boundaries I. Calerkin (spectral) representations. Stud. appl. math., № 4(50), pp. 293-327.

11. Orszag, S.A. (1971). Galerkin approximations to flows within slabs, spheres and cylinders. Phys. rev. lett., № 18(26), pp.1100-1103.

12. Orszag, S.A. (1971). Accurate Solution of the Orr-Sommerfeld stability equation. J. fluid mech., № 4(50), pp. 689-701.

13. Ku, H.G., \& Hatziavramidis, D. (1985). Solutions of the two-dimensional Navier Stokes equations by Chebyshev expansion methods. Computers and fluids, № 1(13), pp. 99113.

14. Fulton, S.R., \& Taylor, G.D. (1984). On the Gottlieb-Turkel time filter for Chebyshev spectral methods. J. comput. phys., № 2(55), pp. 302-312.

15. Gottlieb, D. (1981). The stability of pseudospectral-Chebyshev methods. Math. comput., № 153(36), pp. 107-108.

16. Igumnov, A.B., \& Solov'ev, A.S. (1986). Ustojchivyj psevdospektral'nyj metod reshenija nachal'no-kraevyh zadach. Zh. vychisl. matem. $i$ matem. fiz., T. 26, № 12, pp. 1895-1900.

17. Igumnov, A.B., Solov'ev, A.S., \& Janenko, N.N. (1983). Chislennoe izuchenie volnovyh javlenij $\mathrm{v}$ nelinejnyh sredah $\mathrm{s}$ dispersiej. (p.25). Novosibirsk. (Preprint / AN SSSR Sib. otd-nie. In-t teoret, i prikl. mehaniki; № 25 - 83).

18. Patera, A.T. (1984). A spectral element method for fluid dynamics: laminar flow in a channel expansion. J. Comp. Phys., V. 54, pp. 468-488. 